\title{
Spinal trauma
}

\author{
Jim Ellis • Ron Courson • Brian Daniels
}

Published online: 17 September 2014

(C) Springer Science+Business Media New York 2014

\begin{abstract}
The practice of spinal immobilization has existed since the 1960s under the premise that trauma victims with cervical spine injuries may suffer neurologic injury if moved without stabilization consisting of a rigid cervical collar and long spine board. Because of this assumption, it is of particular importance to assess for movement of the cervical spine with and without spinal immobilization. Over time, the on-field management of athletes with a mechanism consistent with spinal cord injury (SCI) has evolved and produced protocols that can be considered standard of care. Attempts to find evidencebased research to verify the necessity of a rigid collar and long spine board as the only option in athletic medicine for suspected SCI is difficult. As changes occur in the Emergency Medical Services standards, there will be opportunities to see how their processes relate to athletes and the rationale for immobilization on the field of play. Going forward, there could very well be a significant change in the approach to and management of the athlete down on the field of play with a suspected spinal cord injury.
\end{abstract}

Keywords Spinal trauma $\cdot$ Sports injuries $\cdot$ On-field management $\cdot$ Athletic medicine $\cdot$ Emergency care of athlete .

\section{J. Ellis $(\square)$}

Steadman Hawkins Clinic of the Carolinas, Greenville Health

System, Greenville, SC, USA

e-mail: JEllis@ghs.org

\section{J. Ellis}

Clinical Emergency Medicine, Department of Orthopedic Surgery,

University of South Carolina School of Medicine - Greenville,

7 Independence Pointe, Suite 100, Greenville, SC 29615, USA

\section{R. Courson}

Sports Medicine, University of Georgia, Athens, GA 30603, USA

B. Daniels

ProHEALTH Care Associates, Lake Success, NY 11042, USA
Spinal immobilization - EMS protocols for cervical spine injuries $\cdot$ Spinal cord injury

\section{Introduction}

Although it is difficult to substantiate in the literature, the assumption is that Emergency Medical Services (EMS) and Advanced Trauma Life Support (ATLS) have been the key drivers in the on-field management of athletes with suspected spinal trauma. Understandably, they have been used as the gold standard of evaluation and management in the prehospital setting in the United States (US). Many studies have attempted to assess movement of the cervical spine during immobilization, during helmet removal, and during move-

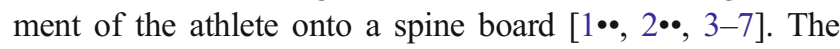
emergency medicine community has taken a critical look at the rationale for routine immobilization of the trauma patient without considering specific mechanisms of injury and patient symptoms $[8 \bullet \bullet, 9 \bullet, 10 \bullet]$. A number of EMS agencies are changing their approach to the trauma patient and there needs to be a question as to how that will change the treatment of the athlete on the field of play. The National Athletic Trainers' Association (NATA) recently published a statement on these EMS changes and the potential impact in athletic medicine $[11 \bullet \bullet]$.

Currently, the medical personnel providing coverage at sporting events in the US varies from youth sports to high school, college, and professional staffing models. Youth sports and some high schools may not have athletic trainers (ATCs) or EMS coverage at events, even the highest risk sports like football and hockey. Some states have enacted laws requiring athletic trainers at high school football games and many high schools have EMS coverage at football games only. All 4 major US professional leagues have ATCs, team physicians, and EMS coverage required at their games. The 
overwhelming majority of colleges have the same coverage, at least for football. Medical coverage at practices also may vary in personnel at each level as 1 high school ATC may be covering 4-5 practices at the same time. This variability in coverage and utilization of EMS can lead to mismanagement of injuries if the issues are not clearly addressed in a unified approach by all stakeholders in the arena of sports medicine and emergency care [12•].

The NATA has a long history of evaluating the up to date literature and releasing position statements on the management of athletes with suspected spinal trauma $[2 \bullet \bullet, 11 \bullet, 13 \bullet]$. Their recently released statement on the changes in EMS protocols could result in a significant change to the approach of the athlete with suspected spinal trauma [11••]. Most medical personnel participating in team coverage follow those guidelines. At the highest levels of competition, athletic trainers, team physicians, and EMS professionals form a unified triad and work together as a team in the on-field management of the athlete with suspected spinal trauma. The preparation for this incident should be clearly defined in the written Emergency Action Plan (EAP) [14•]. Specific roles for each member of the medical team are delineated and this should be practiced at least once a year with all members of the medical team in attendance and working together to practice immobilizing a patient. As the changes in the EMS approach to the suspected spinal trauma patient evolve, it is imperative that everyone involved in the emergency care of the athlete understand the science and rationale for change.

\section{Historical perspective}

Spinal immobilization became standard practice before it was formally studied [15••]. In 1967, at the annual session for the American Association for the Surgery of Trauma, backboards were advocated for their use in the extraction of accident victims [16]. Spinal immobilization gained further attention after Bohlman reported on 300 cases of cervical spine injuries from 1950 through 1972, where he concluded that there was spinal deterioration in some patients after neck immobilization was not provided [17]. Later studies also demonstrated that there was a significant increase in the number of traumatized patients being admitted with preserved neurologic function after more widespread use of pre-hospital spinal immobilization [18]. More recent reviews, however, have failed to demonstrate any new reported cases of spinal cord injury in the course of normal patient handling, leading to the conclusion that many of the earlier reports were more anecdotal [19•, 20].

In the years after Bohlman's paper, spinal immobilization became the standard of care. Beginning in 1971, the American Academy of Orthopedic Surgeons recommended spinal immobilization for patients with symptoms and physical findings of potential spinal cord injury [20]. In the next 2 decades, though, there was a shift from providing spinal immobilization in the pre-hospital setting based on a clinical evaluation to providing immobilization based on the mechanism of injury $[20,15 \cdot 0]$. Since then, however, the pendulum started swinging back toward the use of clinical criteria based on a patient's symptoms and physical examination with continued consideration of the mechanism of injury [20-23]. Today, members of the team medical staff still immobilize the spine as a standard of care, but there is more focus on whether or not to immobilize the spine due to the potential complications associated with it [24, 25].

The rationale for spinal immobilization is not based on any randomized trials, but based on anatomic and mechanical considerations. An older Cochrane review does state that there is insufficient evidence for spinal immobilization and the American Association of Neurological Surgeons also state that there is no Class I or II evidence that recommend any current guidelines $[26 \bullet \bullet, 27 \bullet]$. However, the EMS and ATLS guidelines still support spinal immobilization for patients with suspected spinal cord or vertebral injury, however [23, 28]. A rigid spine board and cervical collar are the currently recommended devices to immobilize the spine until spinal injury can be excluded [23].

Most of these recommendations have been based on accident victims, without a specific focus on the athletic population. Athletes have inherent circumstances specific to them. Athletic equipment removal has been a source of controversy while trying to provide spinal immobilization. Also, athletes and providers alike need more information about return to play. In 1998, the National Athletic Trainers' Association formed an inter-association task force to develop guidelines for the care of athletes with suspected spinal cord injuries. Until that time, there was no clear consensus on the care of injured athletes. This article also acknowledged the circumstances specific to athletics including equipment removal, injuries and possible mechanisms, as well as return to play criteria [13•]. Since that time, there has been a lot of focus on spinal cord injury and spinal immobilization in the athlete.

\section{Current recommendations}

The current approach to the athlete down on the field of play is to assume spinal cord injury until proven otherwise. If mechanism of injury is observed by a member of the medical staff consistent with the potential for spinal trauma, then the initial approach is to stabilize the head and spine on arrival to the patient. If the player obviously exhibits a decreased level of consciousness, has a complaint of neck pain, is tender in the cervical spine with palpation or has any evidence of neurologic compromise including complaints of peripheral paresthesia, numbness, weakness, or radicular pain then the decision to immobilize the patient should proceed. The initial 
approach to stabilize the head with 1 member of the team should be defined in the Emergency Action Plan so there is no confusion in the role of each individual on the medical team [14 $]$. At this point, a more detailed physical exam should be undertaken to evaluate the neurologic status of the patient/ athlete.

The decision to immobilize or not immobilize the athlete is typically made by the highest level and/or most appropriate provider evaluating the patient — physician, athletic trainer, or paramedic/EMT. When multiple providers are present, the decision maker should be designated in advance. This should be clearly spelled out in the EAP so there is no disagreement among providers on the field of play. Once the decision to immobilize is made, the team designated to undertake this procedure is activated as part of the EAP. Practice of the EAP and spinal immobilization should be undertaken in the preseason so the plan will flow smoothly if activated in practice or a game day scenario. The acceptable procedures include log rolling a prone athlete, log roll for a supine athlete, lift and slide of a supine athlete, and a $6+$ person lift for a supine athlete [29]. It is recommended that at least 3 rescuers and preferably 5-6 are available to log roll the player and that at least 5 rescuers and preferably 6 are available for the lift-andslide procedure.

The medical team member designated to be at the head will control c-spine stabilization and will be in charge of voicing commands in the log roll or lift-and-slide maneuvers and long spine board immobilization. Once the athlete is in the proper position on the long spine board, the patient's torso and legs should be secured with spider straps or speed clips (if speed clips, then 7 straps are recommended: 2 should go across the chest from shoulder to opposite axilla, one should go across chest under axilla, 1 should go across the abdomen, 1 should go across the pelvis, 1 should go across the mid thighs and 1 should go across the mid tibias). The athlete's arms should be left free from straps in order to facilitate vital sign monitoring and IV access as needed. The athlete's wrists should be secured in front of the body with a Velcro strap or tape once the patient is secured to the long-spine board. After the torso and legs are secured, the helmet/head should be secured last. If necessary, padding can be placed under the athlete's head to fill in any voids and maintain a neutral in-line spinal position. The head should be stabilized with lateral restraint pads and afterwards secured to the long-spine board with tape over the forehead and at the chin. After the athlete has been properly secured to the long-spine board, the neurologic status should be thoroughly reassessed.

To reiterate, the decision as to which procedure should be utilized needs to be decided and practiced in the preseason EAP scenario session. All helmeted athletes should have the facemask removed for unencumbered access to the airway. Most providers still follow the recommendation for athletes with both helmet and shoulder pads to leave them on during spinal immobilization to keep the spine in a neutral position. If the expertise exists and with a thorough understanding of the correct procedure, it is acceptable to remove both helmet and shoulder pads on the field of play prior to immobilization. The time spent immobilized on the spine board directly correlates with complications such as skin breakdown, pressure sores, aspiration pneumonia, and possibly increased intracranial pressure $[30,31 \bullet]$. This stands to remind us that spinal immobilization is a procedure not without significant morbidity, and one which must be evaluated to make sure it is necessary.

If EMS is on scene when the injury occurs, they are a very important part of the medical team in the care of the athlete. Without on-site EMS, a 911 call should be initiated as soon as the decision is made to immobilize the athlete. If integrated as part of the team, they can help with lifting and can also provide airway expertise in the event of respiratory compromise, which can be seen with high cervical spine injury and also with concomitant spinal cord injury and concussion. In the event of an airway emergency, it is not recommended to proceed with helmet removal as it is more time consuming and the provider should immediately remove the facemask [31•, $32 \bullet \cdot$. With the changes in EMS protocols on spinal trauma, this makes it even more important to practice the EAP in the preseason with EMS personnel. While they can lend knowledge and experience in this setting, the athlete is best known by team physicians and ATCs, so they should take the lead role in managing the athlete with suspected spinal cord injury. Many emergency departments do not have expertise in helmet and shoulder pad removal prior to imaging, so it is recommended that if a member of the medical staff is available, they should accompany the athlete to help with this process at the institution.

Although the number of catastrophic cervical spine injuries in sports is decreasing, the recommended destination for transport of an athlete that has been immobilized should be to the nearest APPROPRIATE facility [32••]. Sports related spinal cord injury constitutes $8.9 \%$ of the total cases [33]. If a Level I Trauma Center is available, that institution should be the primary receiving facility in the EAP for the patient with a suspected spinal cord injury. Exceptions to this recommendation would include: no designated trauma center, unstable airway that would dictate going to the nearest Emergency Department (ED), and a hospital that has a center of excellence in the treatment of patients with spinal cord injury. Again, this receiving facility should be clearly designated in the EAP and there should be a specific receiving physician that can care for the athlete with the ability to perform rapid surgical decompression if appropriate. There have been a number of treatments researched in the literature for care of the athlete with spinal cord injury (steroids, cold intravenous fluids) but the definitive procedure of decompression has shown to produce the best outcomes [34]. The amount of time to get the patient in the operating room is a critical predictor of 
a successful outcome and needs to be an area of focus in this specific scenario for the athlete.

\section{Future considerations}

There is currently ongoing significant change to protocols of Emergency Medical Response Services in some parts of the country $[11 \bullet \cdot$. The change affects how EMS will be transporting patients to the Emergency Department. In many cases of suspected spine injury, EMS might not recommend immobilizing a patient to a spine board for transport. Rather, patients may be transported with a cervical collar only and secured directly to the stretcher for transport. These recent developments have placed new emphasis on considerations for the possibility of removing football equipment on the field of play with suspected head and/or spine Injuries [35••]. Although every athletic injury situation may not be appropriate, based upon individual circumstances, there are times when the athlete may be best treated by on-site equipment removal. Thus, it is critically important to review and rehearse appropriate techniques. Rationale for consideration of equipment removal in the field is based upon 4 concepts:

- Advances in equipment technology: changes in helmet and shoulder pad design have helped to facilitate equipment removal.

- Equipment removal performed by those with highest level of training: On the field of play there may be multiple individuals with knowledge of equipment removal vs inexperienced Emergency Department personnel.

- Better packaging: with helmet and shoulder pads removed, the athlete is in a spine neutral position. A cervical collar may be applied. Packaging straps are closer to body with equipment removed.

- Expedited care in ED: with equipment removed prior to arrival in ED, physician evaluation and diagnostic tests may be expedited.

Consideration should be given to the possibility of equipment removal on site prior to transport, where more rescuers familiar with equipment removal may be available vs the emergency facility and the athlete may be better immobilized without equipment as a barrier. Removal of helmet and shoulder pads on site is appropriate when:

- The helmet is not properly fitted to prevent movement of the head independent of the helmet;

- the equipment prevents neutral alignment of the cervical spine;

- the equipment prevents airway or chest access; or

- the trained rescuers on site feel, based upon the individual circumstances, on site equipment removal is indicated.
The following general principles should be applied when helmet and shoulder pad removal are decided to be the most appropriate course of action:

- Remove helmet first;

- next, remove shoulder pads;

- next, apply cervical collar;

- then, secure to spine board with straps to body first, incorporating $\mathrm{x}$-strap technique (shoulder/axilla) to minimize cephalo-caudal movement on board;

- make sure to keep arms free, secure wrists;

- finally, secure head last with head immobilization device.

The helmet and shoulder pad removal are best accomplished with the entire medical team, ideally 9 trained personnel. The best practice technique includes first removing the helmet in the technique described below:

- rescuer 1 stabilizes c-spine;

- rescuer 2 assume c-spine control from front, allowing rescuer 1 to release;

- rescuer 1 removes helmet; then again assumes c-spine control, allowing rescuer 2 to release;

- lift and slide: 9 person technique: rescuer 1 stabilizes cspine; jersey and shoulder pads cut in front; rescuers 2-7 ( 3 on each side) lift athlete 12" on command (to allow shoulder pad clearance for removal); rescuer 8 slides board in; Rescuer 9 removes shoulder pads; athlete lowered to board on command.

If the helmet is removed, spinal immobilization must be maintained while removing. Dependent upon the type of football helmet worn, it may be appropriate to remove cheek padding and/or deflate air padding prior to helmet removal. Shoulder pads do not necessarily have to be removed on site. The front of the shoulder pads can be opened to allow access for CPR and defibrillation. Should either the helmet or shoulder pads be removed - or if only 1 of these is presentappropriate spinal alignment must be maintained. A recent study evaluates the pack and fill protocol as another technique that may be considered. $[1 \bullet \bullet$.

\section{Conclusions}

More research, along with educational initiatives, is needed in the area of emergency care, particularly related to equipment intensive sports, from both a clinical practice perspective and an athletic specific research initiative. Sports medicine utilizes the team concept, with health care professionals working together. By facilitating communication and education among athletic trainers, paramedics, emergency physicians, and team physicians, we will ultimately improve patient care in this 
critical setting. It is recommended that athletic health care providers conduct a "Time-Out" before athletic events to ensure emergency action plans are reviewed and in place [36••]. "Time-Out" is a common term both in sports and medicine. Coaches and athletes call time-outs to gather a team together and discuss game strategies or to call a play. In medicine, doctors take a time-out immediately before every surgery when all operating room participants stop to verify the procedure, patient identity, correct site, and side. This brief review of the emergency action plan and discussion among athletic health care team members beforehand helps to ensure that a coordinated emergency approach is in place.

The care of the athlete with suspected spinal trauma has evolved over the last 50 years. As we see changes in the athletic medicine literature and with EMS protocols, team physicians and ATCs need to be aware of the information and make decisions that are evidence based. These protocols may significantly change the approach to the athlete with suspected spinal cord injury and therefore, the procedures in how we care for the athlete. Going forward, it is imperative to keep up with the evidence based changes and incorporate them in to the Emergency Action Plan written and practiced at each venue where athlete care occurs.

\section{Compliance with Ethics Guidelines}

Conflict of Interest Jim Ellis, Brian Daniels and Ron Courson declare that they have no conflict of interest.

Human and Animal Rights and Informed Consent No human or animal studies performed by the authors:

This article does not contain any studies with human or animal subjects performed by any of the authors.

Human studies done by authors (but no animal studies)

This article does not contain any studies with animal subjects performed by any of the authors. With regard to the authors' research cited in this paper, all procedures were followed in accordance with the ethical standards of the responsible committee on human experimentation and with the Helsinki Declaration of 1975, as revised in 2000 and 2008.

Animal studies done by authors (but no human studies)

This article does not contain any studies with human subjects performed by any of the authors.

With regard to the authors' research cited in this paper, all institutional and national guidelines for the care and use of laboratory animals were followed.

\section{References}

Papers of particular interest, published recently, have been highlighted as:

- Of importance

-. Of major importance

1.• Jacobson B, Cendoma M, Gdovan J, Cooney K, Bruening D. Cervical spine motion during football equipment-removal protocols: a challenge to the all or nothing endeavor. J Athl Train.
2014;49:42. Recent article that challenges what most consider the standard of care.

2.• Swartz E, Boden B, Courson R, Decoster L, Horodyski M, Norkus S, et al. National Athletic Trainers' Association Position Statement: Acute Management of the Cervical Spine-Injured Athlete. J Athl Train. 2009;44:306-31. Considered the standard of care by most in athletic medicine.

3. Shrier I, Boissy P, Brire S, Mellette J, Fecteau L, Matheson G, et al. Can a rescuer or simulated patient accurately assess motion during cervical spine stabilization practice sessions? J Athl Train. 2012; 47:42.

4. Kordecki M, Smith D, Hoogenboom B. On the sidelines: a clinical suggestion; the Riddell RipKord System for shoulder pad removal in a cervical spine injured athlete: a paradigm shift. Int J Sports Phys Ther. 2011;6:142.

5. Decoster LC, Burns MF, Swartz EE, Murthi DS, Hernandez AE, Bailas JC, et al. Maintaining neutral sagittal cervical alignment after football helmet removal during emergency spine injury management. Clin J Sport Med. 2010;6:436-44.

6. Swartz E, Rossi G. Cervical spine alignment during on-field management of potential catastrophic spine injuries. Sport Health: Multidiscipl Approach. 2009;1:247.

7. Treme G, Diduch DR, Hart J, Romness MJ, Kwon MS, Hart JM. Cervical spine alignment in the youth football athlete: recommendations for emergency transportation. J Athl Train. 2008;1:14-20.

8.• Hauswald M. A re-conceptualisation of acute spine care. Emerg Med J. 2013;9:720-3. A more recent evaluation of whether or not we routinely need to immobilize spinal trauma patients.

9. Hauswald M, Tandberg D, Omar Z. Out-of-hospital spinal immobilization: its effect on neurologic injury. Acad Emerg Med. 1998;5: 214-9. One of the better articles to question whether or not it was necessary to immobilize spinal trauma patients.

10. McHugh TP, Taylor JP. Unnecessary out-of-hospital use of spine immobilization. Acad Emerg Med. 1998;5:278-80. Another article evaluating unnecessary immobilization.

11.• National Athletic Trainers' Association Official Statement: EMS Changes to Pre-hospital Care of the Athlete with Acute Cervical Spine Injury. 2014. Very important recent statement by the NATA on the changes in the approach to the spinal trauma patient by EMS agencies.

12. Decoster L, Swartz E, Cappaert T, Hootman J. Prevalence and characteristics of general and football-specific emergency medical service activations by high school and collegiate certified athletic trainers: a national study. Clin J Sport Med. 2012;20:436. Nice study looking at how EMS is activated by athletic trainers at different levels.

13. Kleiner DM, Almquist JL, Bailes J, Burruss P, Feuer H, Griffin LY, et al. Prehospital care of the spine-injured athlete: a document from the Inter-Association Task Force for Appropriate Care of the SpineInjured Athlete. Dallas, TX: National Athletic Trainers' Association; 2001. Felt by many people to be some of the best work on care of the athlete with spinal injury as put together by the landmark task force assembled.

14. Anderson J, Courson R, Kleiner D, McLoda T. National Athletic Trainers' Association Position Statement: Emergency Planning in Athletics. J Athl Train. 2002;1:99-104. The EAP is the basis for the approach to the seriously ill or injured athlete and this addressed the proper planning to prepare for this.

15.• Manoach S, Paladino L. Manual in-line stabilization for acute airway management of suspected cervical spine injury: historical review and current questions. Ann Emerg Med. 2007;50:236-45. Very good review of airway management in the spinal injured patient.

16. Farrington JD. Extraction of victims - surgical principles. J Trauma. 1968;8:493-512. 
17. Bohlman HH. Acute fractures and dislocations of the cervical spine. An analysis of three hundred hospitalized patients and review of the literature. J Bone Joint Surg Am. 1979;61:1119-42.

18. Crosby ET. Tracheal intubation in the cervical spine-injured patient. Can J Anaesth. 1992;39:105-9.

19. Ahn H, Singh J, Nathens A, et al. Pre-hospital care management of a potential spinal cord injured patient: a systematic review of the literature and evidence-based guidelines. J Neurotrauma. 2011;28: 1341-61. Good review of the literature and evidence-based guidelines on the spinal injured patient.

20. Domeier RM. Indications for prehospital spinal immobilization. National Association of EMS Physicians Standards and Clinical Practice Committee. Prehosp Emerg Care. 1999;3:251-3.

21. Hoffman JR, Mower WR, Wofson AB, Todd KH, Zucker MI. Validity of a set of clinical criteria to rule out injury to the cervical spine in patients with blunt trauma. N Engl J Med. 2000;343:94-9.

22. Stiell IG, Wells GA, Vandemheen K, et al. The Canadian C-Spine Rule for radiography in alert and stable trauma patients. JAMA. 2001;286:1841-8.

23. American College of Surgeons Committee on Trauma. Advanced trauma life support-student course manual. 9th ed. Chicago: American College of Surgeons; 2012.

24. Kwan I, Bunn F. Effects of prehospital spinal immobilization: a systematic review of randomized trials on healthy subjects. Prehosp Disaster Med. 2005;20:47-53.

25. Stuke LE, Pons PT, Guy JS, Chapleau WP, Butler FK, McSwain NE. Prehospital spine immobilization for penetrating trauma-review and recommendations from the Prehospital Trauma Life Support Executive Committee. J Trauma. 2011;71:763-9.

26.• Theodore N, Hadley MN, Aarabi B, et al. Prehospital cervical spine immobilization after trauma. Neurosurgery. 2013;72:22-34. Very well done review stating that there are no Class I or II recommendations for any guidelines in spinal trauma on immobilization.

27. Kwan I, Bunn F, Roberts I. Spinal immobilisation for trauma patients. Cochrane Database Syst Rev. 2001;2, CD002803. Earlier study than above that found the same lack of evidence.
28. U.S. Department of Transportation, National Highway Traffic Safety Administration. National Emergency Medical Services education standards-Emergency Medical Technician instructional guidelines. Washington, DC: Government Printing Office; 2009.

29. Del Rossi G, Horodyski MH, Conrad BP, Di Paola CP, Di Paola MJ, Rechtine GR. The 6-plus-person lift transfer technique compared with other methods of spine boarding. Curr Sports Med Rep. 2007;6:32-5.

30. Beattie L, Choi J. Acute spinal injuries: assessment and management. J Emerg Med Pract. 2006;5:1-4.

31. Bhamra JS, Morar Y, Khan WS, Deep K, Hammer A. Cervical spine immobilization in sports related injuries:review of current guidelines and a case study of an injured athlete. Open Orthopaed J. 2012;6:548-52. Sports specific review of guidelines on the athlete with spinal injury.

32.• Swartz E, Mihalik J, Beltz N, Day M, Decoster L. Face mask removal is safer than helmet removal for emergent airway access in American Football. Spine J. 2014;14:996-1004. Another good article addressing airway emergencies and comparing the fastest method to secure the airway especially in light of the changes suggesting on-field helmet removal.

33. Cantu RC, Li YM, Abdulhamid M, Chin LS. Return to play after cervical spine injury in sports. Curr Sports Med Rep. 2013;1:14-7.

34. Assenmacher B, Schroeder G, Patel A. On-field management of spine and spinal cord injuries. Oper Tech Sports Med. 2013;21: 152.

35.• Reifsteck F, Walpert K, Courson R, Henry G. University of Georgia Sports Medicine Spine Injury Management Protocol. 2012. Excellent protocol for dealing with the athlete suspected of having a spinal cord injury outlining approach and steps in immobilization.

36.• Courson R, Mandelbaum B, Lemak L. Adopt a "Time-Out" system before sporting events. National Athletic Association Statement. 2012. Simple but excellent suggestion to take time to refresh everyone's mind on the medical team before an event and review the EAP. 